\title{
A comparison of the effects of oral nadolol and topical timolol on intraocular pressure, blood pressure, and heart rate
}

\author{
GEOFFREY R DUFF,' ANDREW H WATT,' AND PETER A GRAHAM' \\ From the 'Department of Ophthalmology, University Hospital of Wales, and the 'Department of Pharmacology \\ and Therapeutics, University of Wales College of Medicine, Cardiff
}

\begin{abstract}
SUMMARY We compared the effects of oral nadolol $20 \mathrm{mg}$ once daily and topical timolol $0.25 \%$ twice daily on the intraocular pressures of 22 glaucoma suspects, in a 12 week double-masked crossover study. Both nadolol and timolol reduced intraocular pressure $(p<0 \cdot 001)$. The reductions in intraocular pressure did not differ significantly after two weeks and four weeks of treatment. Nine of these subjects underwent treadmill exercise tests in order to compare the effects of nadolol and timolol on the cardiovascular system. The effects of the two treatments on blood pressure were comparable, but nadolol caused a significantly greater reduction in heart rate than timolol at rest $(p<0.05)$. Nadolol reduces intraocular pressure effectively and may prove a useful alternative treatment for chronic simple glaucoma.
\end{abstract}

Introduced in 1979 for the treatment of chronic simple glaucoma, timolol offered a relatively low frequency of local side effects when compared with established topical treatment. The ease of administration of a long-acting oral beta blocking agent might further improve patient compliance. Nadolol is a non-selective beta blocker with a plasma half-life of 17-23 hours. It reduces intraocular pressure over a 24-hour period when applied topically to the eye in a $2 \%$ solution, ' but habituation appears to occur within one month. ${ }^{2}$ When administered systemically in doses of $10-80 \mathrm{mg}$ once daily it reduces intraocular pressure,${ }^{34}$ and a reduction can be obtained for at least 24 months on long-term therapy. ${ }^{5}$ We compared the effects of oral nadolol and topical timolol, on intraocular pressure, blood pressure, and heart rate in a 12-week double-masked crossover study.

\section{Patients and methods}

Twelve male and 12 female patients, aged 47-78, with normal visual fields were recruited from a glaucoma clinic. These patients were thought to be at risk of developing glaucoma because of their intraocular

Correspondence to G R Duff, Department of Ophthalmology, UHW, Heath Park, Cardiff CF4 4XW. pressures, a family history, or the appearance of their optic discs. Exclusion criteria were: confirmed open angle or closed angle glaucoma, any visual field defect, visual acuities of less than $6 / 12$, corneal disease, a history of ocular surgery, asthma, chronic obstructive airways disease, cardiac failure, renal dysfunction, or current therapy with a beta blocking agent.

Ethical approval was obtained from the hospital ethics committee. All subjects gave written informed consent and underwent a pretrial physical examination (including an electrocardiogram for those subjects undergoing exercise tests). All subjects had their visual fields checked with a Goldmann perimeter (Armaly technique ${ }^{\text {) }}$ to ensure that they were normal. Those on ocular hypotensive treatment had their case histories and visual fields reviewed by an independent observer before their medication was stopped at least two weeks prior to the trial.

This double-masked crossover study lasted 12 weeks. Of 24 subjects entered in the trial one was withdrawn because of an electrocardiographic abnormality found in preparation for the exercise test, and one was withdrawn because of malaise and lethargy while on timolol. The subjects were randomly allocated into two groups. There were 13 subjects in group 1 and nine in group 2 . Throughout 
the trial the subjects took one tablet once a day, and instilled one drop twice a day in each eye. Group 1 received nadolol $20 \mathrm{mg}$ tablets once daily and placebo drops twice daily for four weeks, followed by a fourweek wash-out during which they received placebo drops and placebo tablets. After crossover they received timolol $0.25 \%$ drops twice daily and placebo tablets once daily for four weeks. Group 2 was started on timolol drops (and placebo tablets) for four weeks, and after a four-week wash-out and crossover they continued with nadolol tablets (and placebo drops).

Intraocular pressures were measured at baseline and then fortnightly for 12 weeks. All the intraocular pressure measurements were made within 10 hours of dosage by the same investigator (GRD) at the same time of day for each subject (to within 25 minutes). Subjects had their visual fields reassessed at the end of the trial if their intraocular pressures rose to levels higher than those found at the time of their referral to the glaucoma clinic.

The cardiovascular effects of nadolol and timolol were studied, and 10 subjects underwent symptomlimited treadmill exercise tests on three occasions: off treatment, on nadolol, and on timolol. One of the 10 subjects was unable to complete the treadmill tests as she sustained a back injury at home. There were five subjects in treatment group 1 and four in treatment group 2. The duration of the test off treatment was taken as the end point of further tests during each treatment period. Heart rate and blood pressure data immediately after peak exercise are reported.

Log transformation of the data on intraocular pressures helped to reduce the skew towards zero, and the analyses are therefore based on log transformed data. The averages of two eyes are used in order to minimise imprecision. There is no consistent evidence of skew in the cardiovascular data such as would suggest the need for a $\log$ transformation.

The statistical significance of the changes in intraocular pressure, blood pressure, and heart rate on each treatment was assessed by paired $t$ tests comparing values on treatment with preceding baseline values (that is, the readings at 0 or 8 weeks).

The comparison of changes in intraocular pressure, blood pressure, and heart rate between the two treatments was performed by the method of Hills and Armitage. ${ }^{7}$ The difference in effect between treatments was assessed by an unpaired $t$ test comparing period differences in the two random groups (group 1: nadolol first, $n=13$; group 2: timolol first, $n=9$ ), with corresponding analyses for period and interaction effects. However, when the efficacy of the washout period was examined by paired $t$ tests, the intraocular pressure results for right eyes after four weeks of washout were significantly higher than at week $0 \quad(\mathrm{p}<0.05)$. The intraocular pressure crossover comparisons were therefore more validly based on increments from the preceding baseline values. When this was done there was no evidence of corruption by period or interaction effects. Confidence intervals are given for the difference in effect between treatments.

\section{Results}

INTRAOCULAR PRESSURES

Table 1 summarises the distribution of intraocular pressure before treatment (weeks 0 and 8 ) and after two and four weeks of treatment with timolol and nadolol. Appropriate summary measures for the log normal distribution are given, the geometric mean, and fitted 2.5 and 97.5 centiles. Both treatments produced a highly significant reduction in intraocular pressure $(p<0.001)$. There was no significant evidence of any difference in effectiveness between the treatments. After two weeks of treatment the values on nadolol were $+13 \%$ higher than on timolol, taking baseline values into account. A $95 \%$ confidence interval for this difference is from $-13 \%$ to $+47 \%$. The corresponding difference at four weeks was $+8 \%$, with a $95 \%$ confidence interval from $-3 \%$ to $+36 \%$.

Table 1 Summary statistics for intraocular pressure ( $\mathrm{mmHg}$ ), off treatment, on timolol and on nadolol; based on 22 subjects, eyes averaged

\begin{tabular}{llll}
\hline & $\begin{array}{l}\text { Geometric mean } \\
\text { IOP }\end{array}$ & \multicolumn{2}{l}{$\begin{array}{l}\text { Fitted 2.5 } \\
\text { centiles }\end{array}$} \\
\hline Off treatment, weck 0 & 22.5 & 13.6 & 37.3 \\
Off treatment, weck 8 & 21.9 & 12.6 & 37.3 \\
Aftcr 2 wceks' timolol & 16.5 & 11.2 & 24.5 \\
After 4 wecks' timolol & 16.9 & 10.1 & 26.5 \\
Aftcr 2 wceks' nadolol & 17.7 & 10.1 & 30.7 \\
After 4 weck' nadolol & 17.7 & 10.8 & 29.1 \\
\hline
\end{tabular}

HEART RATE AND BLOOD PRESSURES

Table 2 summarises the haemodynamic effects in the nine patients tested. On nadolol the heart rates at rest and after exercise were greatly reduced. The corresponding reduction on timolol was approximately one-third as great and did not reach statistical significance. The reduction in resting heart rate on nadolol was significantly greater than that observed on timolol $(p<0.05)$; the corresponding difference after exercise approached significance.

The two drugs produced comparable effects on blood pressure; with a decrease in systolic blood pressure, most marked after exercise. 
Table 2 The haemodynamic effects of nadolol and timolol in nine subjects. Serial changes from baseline values are assessed by paired tests

\begin{tabular}{|c|c|c|c|c|c|}
\hline & \multirow{2}{*}{$\begin{array}{l}\text { At baseline } \\
\text { (SD) }\end{array}$} & \multirow{2}{*}{$\begin{array}{l}\text { Onnadolol } \\
(S D)\end{array}$} & \multirow{2}{*}{$\begin{array}{l}\text { On timolol } \\
(S D)\end{array}$} & \multicolumn{2}{|l|}{ Crossover analysis } \\
\hline & & & & $\begin{array}{l}\text { Mean difference } \\
\text { nadolol minus timolol }\end{array}$ & ( $95 \%$ confidence interval) \\
\hline \multicolumn{6}{|c|}{ Heart ratc, bpm } \\
\hline Rest & $79(12)$ & $57(7)^{* *}$ & $73(11)$ & -16 & $(-27$ to -6$)$ \\
\hline Exercisc & $110(27)$ & $81(15)^{*}$ & $101(23)$ & -20 & $(-40$ to +1$)$ \\
\hline \multicolumn{6}{|c|}{ Systolic blood pressurc, $\mathrm{mmHg}$} \\
\hline Rest & $147(22)$ & $137(23)$ & $133(22)$ & +3 & $(-13$ to +19$)$ \\
\hline Exercise & $181(20)$ & $156(19)^{* * *}$ & $163(11)^{* * *}$ & -9 & $(-21$ to +4$)$ \\
\hline \multicolumn{6}{|c|}{ Diastolic blood pressurc, $\mathrm{mmHg}$} \\
\hline Rest & $88(12)$ & $86(12)$ & $86(10)$ & $-0 \cdot 2$ & $(-8$ to +8$)$ \\
\hline Exercise & $94(13)$ & $85(7)$ & $87(12)$ & -3 & $(-12$ to +7$)$ \\
\hline
\end{tabular}

${ }^{* * *} \mathrm{p}<0 \cdot 001,{ }^{* *} \mathrm{p}<0 \cdot 01,{ }^{*} \mathrm{p}<0 \cdot 05$, clsc $\mathrm{p}>0 \cdot 05 . \mathrm{bpm}=$ bcats per minutc.

\section{Discussion}

To our knowledge this is the first controlled study (the subjects acting as their own controls in this crossover study) comparing the effects of oral nadolol and topical timolol on intraocular pressure and on the cardiovascular system. Nadolol produced a significant reduction in intraocular pressure when administered systemically, and this effect is of the same order of magnitude as that produced by timolol. This confirms the results of a previous open study. ${ }^{5}$ In our study the drop in intraocular pressure after treatment with oral nadolol for two weeks and four weeks is very similar to that obtained by Rennie and Smerdon (at days 1 and 8 ). ${ }^{+}$Our observations were all made within 10 hours of dosage, and we are therefore unable to comment on the 24-hour control of intraocular pressure.

The effects of nadolol and timolol on intraocular pressure do not differ significantly, but, if the maximum effects of the two drugs are compared at two weeks, we find that the intraocular pressures on nadolol are $13 \%$ higher than those on timolol. The sample size in this study is not large, and we cannot exclude the possibility of a clinically important difference in the effects of the treatments, but our data are consistent with the two treatments being equally effective.

There is a much greater reduction in heart rate on nadolol, though the effects of nadolol and timolol on blood pressure are similar. This suggests, that, when there is a relative contraindication to the use of beta blocking agents, nadolol will have to be used with caution.

Nadolol is one of a number of beta blocking agents that have been studied with a view to their use in chronic simple glaucoma. The most widely investigated is timolol, which has for some time been established as the first line of treatment. Any proposed alternative glaucoma therapy should at least equal timolol in terms of efficacy, patient compliance, and side effects. In chronic simple glaucoma, whenever beta blockade is not contraindicated and the patients find it difficult or inconvenient to instil drops (or there is some other problem with compliance), nadolol should prove to be a safe and effective treatment.

We are grateful to Dr Newcombe, of the Department of Medical Computing and Statistics, University of Wales College of Medicinc, for his help and advice in the design and analysis of this study.

\section{References}

1 Duzman E, Chi-Chu Chen, Anderson J, et al. Diacetyl derivative of nadolol. I. Ocular pharmacology and short term ocular hypotensive effect in glaucomatous cyes. Arch Ophthalmol 1982; 100: 1916-9.

2 Duzman E, Rosen N, Lazar M. Diacetyl nadolol: 3-month ocular hypotensive effect in glaucomatous eyes. $\mathrm{Br} J$ Ophthalmol 1983; 67: 668-73.

3 Williamson J, Atta HR, Kennedy PA, Muir JG. Effect of orally administered nadolol on the intraocular pressure in normal voluntecrs. BrJ Ophthalmol 1985; 69: 38-40.

4 Rennic IG, Smerdon DL. The effect of a once-daily oral dose of nadolol on intraocular pressure in normal voluntecrs. $\mathrm{Am} \mathrm{J}$ Ophthalmol 1985; 100: 445-7.

5 Williamson J, Young JDH, Atta H, Muir G, Kadom H. Comparative efficacy of orally and topically administered beta blockers for chronic simple glaucoma Br J Ophthalmol 1985; 69: $41-5$.

6 Armaly MF. Selective perimetry for glaucomatous defects in ocular hypertension. Arch Ophthalmol 1972; 87: 518-24.

7 Hills M, Armitage $\mathrm{P}$. The two period cross-over clinical trial. $\mathrm{Br}$ J Clin Pharmacol 1979; 8: 7-20.

Accepted for publication 26 September 1986. 